\title{
Cancer risk associated with chronic diseases and disease markers: prospective cohort study
}

\author{
Huakang Tu, ${ }^{1}$ Chi Pang Wen, ${ }^{2,3,4}$ Shan Pou Tsai, ${ }^{5}$ Wong-Ho Chow, ${ }^{1}$ Christopher Wen, ${ }^{6}$ \\ Yuanqing Ye, ${ }^{1}$ Hua Zhao, ${ }^{1}$ Min Kuang Tsai, ${ }^{2}$ Maosheng Huang, ${ }^{1}$ Colin P Dinney, \\ Chwen Keng Tsao, ${ }^{8}$ Xifeng $\mathrm{Wu}^{1}$
}

${ }^{1}$ Department of Epidemiology,

The University of Texas MD

Anderson Cancer Center,

Houston, TX 77030, USA

${ }^{2}$ Institute of Population Health

Sciences, National Health

Research Institutes, Zhunan,

Taiwan

${ }^{3}$ Graduate Institute of

Biomedical Sciences, College

of Medicine, China Medical

University, Taichung, Taiwan

${ }^{4}$ Department of Medical

Research, China Medical

University Hospital, Taichung,

Taiwan

${ }^{5} \mathrm{MJ}$ Health Research

Foundation, Taipei, Taiwan

${ }^{6}$ Radiology, Long Beach

Veterans Administration

Hospital, University of California

at Irvine, CA, USA

${ }^{7}$ Department of Urology,

The University of Texas MD

Anderson Cancer Center,

Houston, TX, USA

${ }^{8} \mathrm{MJ}$ Health Management

Institution, Taipei, Taiwan

Correspondence to: $\mathrm{XWu}$

xwu@mdanderson.org

Additional material is published online only. To view please visit the journal online.

Cite this as: BMJ 2018;360:k134 http://dx.doi.org/10.1136/bmj.k134

Accepted: 11 December 2017

\section{ABSTRACT}

\section{OBJECTIVES}

To assess the independent and joint associations of major chronic diseases and disease markers with cancer risk and to explore the benefit of physical activity in reducing the cancer risk associated with chronic diseases and disease markers.

DESIGN

Prospective cohort study.

SETTING

Standard medical screening program in Taiwan.

PARTICIPANTS

405878 participants, for whom cardiovascular disease markers (blood pressure, total cholesterol, and heart rate), diabetes, chronic kidney disease markers (proteinuria and glomerular filtration rate), pulmonary disease, and gouty arthritis marker (uric acid) were measured or diagnosed according to standard methods, were followed for an average of 8.7 years.

\section{MAIN OUTCOME MEASURES}

Cancer incidence and cancer mortality.

RESULTS

A statistically significantly increased risk of incident cancer was observed for the eight diseases and markers individually (except blood pressure and pulmonary disease), with adjusted hazard ratios ranging from 1.07 to 1.44 . All eight diseases and markers were statistically significantly associated with risk of cancer death, with adjusted hazard ratios ranging from 1.12 to 1.70 . Chronic disease risk scores

\section{WHAT IS ALREADY KNOWN ON THIS TOPIC}

Studies have shown that certain chronic diseases (eg, diabetes and chronic kidney disease) may predispose to cancer

Investigations generally assessed individual chronic diseases rather than combined

Physical activity has been shown to reduce all cause mortality, but the role of physical activity in reducing the cancer risk associated with chronic diseases is unclear

\section{WHAT THIS STUDY ADDS}

A substantial cancer risk is associated with a combination of cardiovascular disease markers, diabetes, chronic kidney disease markers, pulmonary disease, and gouty arthritis marker

These factors contribute to more than one fifth of incident cancers and more than one third of cancer deaths, comparable to the combined contribution of five lifestyle risk factors

Furthermore, physical activity was associated with a nearly $40 \%$ reduction in the risk of cancer associated with chronic diseases or markers summarizing the eight diseases and markers were positively associated with cancer risk in a doseresponse manner, with the highest scores associated with a 2.21 -fold ( $95 \%$ confidence interval 1.77 -fold to 2.75 -fold) and 4.00 -fold (2.84-fold to 5.63 -fold) higher cancer incidence and cancer mortality, respectively. High chronic disease risk scores were associated with substantial years of life lost, and the highest scores were associated with 13.3 years of life lost in men and 15.9 years of life lost in women. The population attributable fractions of cancer incidence or cancer mortality from the eight chronic diseases and markers together were comparable to those from five major lifestyle factors combined (cancer incidence: $20.5 \%$ v $24.8 \%$; cancer mortality: $38.9 \%$ $v 39.7 \%$ ). Among physically active (versus inactive) participants, the increased cancer risk associated with chronic diseases and markers was attenuated by $48 \%$ for cancer incidence and $27 \%$ for cancer mortality.

\section{CONCLUSIONS}

Chronic disease is an overlooked risk factor for cancer, as important as five major lifestyle factors combined. In this study, chronic diseases contributed to more than one fifth of the risk for incident cancer and more than one third of the risk for cancer death. Physical activity is associated with a nearly $40 \%$ reduction in the cancer risk associated with chronic diseases.

\section{Introduction}

Chronic diseases were responsible for $71 \%$ of deaths globally in 2015, primarily from cancer, cardiovascular disease, diabetes, chronic kidney disease, and respiratory disease. ${ }^{1}$ Most notable were deaths due to cancer, which increased from 5.7 million in 1990 to 8.8 million in $2015,{ }^{12}$ and the global cancer burden is expected to increase by $70 \%$ in the coming two decades. $^{34}$ Cancer and other chronic diseases share common risk factors, including aging and unhealthy lifestyles (eg, smoking, unhealthy diet, physical inactivity, obesity, and alcohol misuse). Certain noncancer chronic diseases may also predispose to cancer independent of their shared risk factors; however, current cancer prevention strategies focus on lifestyle risk factors and do not consider non-cancer chronic diseases as modifiable cancer risk factors, ${ }^{5-7}$ probably because of the modest associations observed.

Diabetes has been consistently associated with a modestly increased risk of cancer. ${ }^{8-10}$ Accumulating evidence has also linked the risk of cancer with cardiovascular disease markers-for example, blood pressure, ${ }^{11-15}$ heart rate, ${ }^{16}{ }^{17}$ total cholesterol level, ${ }^{18-23}$ chronic kidney disease, ${ }^{24-29}$ and gouty arthritis marker (uric acid). ${ }^{30-34}$ In addition, multiple studies 
have implicated pulmonary disease in lung cancer, ${ }^{35}$ but data on other cancer sites are lacking. These studies generally assessed chronic diseases or disease markers individually. As chronic diseases are typically clustered, it is necessary to study them simultaneously to elucidate their independent and joint impact on cancer risk, but few data are available on this topic. We have previously shown that physical activity could reduce all cause mortality, ${ }^{36}$ but the role of physical activity in reducing chronic disease related cancer risk is unclear.

Among 405878 participants of the prospective MJ Cohort study, we investigated the independent and joint effect of several common chronic diseases or disease markers on cancer risk (cancer incidence and cancer mortality). Also, we compared the contribution of chronic diseases or disease markers to cancer risk with that of lifestyle factors. We further assessed whether physical activity could attenuate the cancer risk associated with chronic diseases or disease markers.

\section{Methods}

\section{Study population and data collection}

The details of the MJ Cohort study population and data collection are reported elsewhere. ${ }^{36-38}$ Between 1996 and 2007 the study members participated in a standardized fee-for-service comprehensive medical screening program run by a private firm (MJ Health Management Institution, Taiwan). Self paying participants come from all over Taiwan. Individuals paid by their employers can also participate in the program. There are four geographically representative MJ locations across Taiwan: northern (Taipei), north west (Taoyuan), central (Taichung), and southern (Kaohsiung) parts. The four locations used the same screening protocols and instruments, and data were centralized. The personal characteristics of the participants generally matched those of the general population, except that the cohort is slightly overrepresentative of adults aged less than 40. Participants completed a self administered questionnaire on medical history, lifestyle, and demographic information and underwent a series of medical tests (blood, urine, body measurements, functional tests, and physical examination). Exclusion criteria for the current study were age less than 18 years $(n=14989)$, a history of cancer at the time of recruitment $(n=3574)$, and less than one year of follow-up (31178). The final study cohort consisted of 405878 participants $(48.0 \%$ men and $52.0 \%$ women). The participants were encouraged to return for follow-up visits. Among the 405878 participants, 177430 (43.7\%) had a second visit; the mean time from the baseline visit to the second visit was 2.0 years (SD 1.6 years).

We classified the volume of leisure time physical activity-the product of intensity (metabolic equivalent) and duration of exercise (hours), into five categories: inactive ( $<3.75$ metabolic equivalent (MET) hours /week), low active (3.75-7.49 MET hours/week), medium active (7.50-16.49 MET hours/week), high active (16.50-25.49 MET hours/week), and very high active ( $\geq 25.50$ MET hours/week), with the categories for medium active and above consisting of participants who met the current leisure time physical activity recommendation of 150 minutes a week of moderate intensity exercise or 75 minutes a week of vigorous intensity exercise. ${ }^{3639}$ Total fruit and vegetable intake (servings/day) was the sum of the intake of fruits, deep green vegetables, and light green vegetables.

\section{Chronic diseases and markers}

We selected five common chronic diseases for evaluation, which account for most of the disease burden worldwide: cardiovascular disease (as indicated by diastolic blood pressure and systolic blood pressure, total cholesterol level, and heart rate), diabetes (fasting blood glucose level), chronic kidney disease (dipstick proteinuria and estimated glomerular filtration rate), pulmonary disease (forced expiratory volume in one second and forced vital capacity), and gouty arthritis (uric acid). Box 1 shows the diagnostic criteria or categorization of chronic diseases or markers used in this study.

\section{Follow-up for cancer}

Details of follow-up procedures have been reported previously. ${ }^{36} 37$ Briefly, we obtained information on cancer incidence and vital status by linking study participants to the Taiwan cancer registry and Taiwan death file between 1997 and 2008 using the unique identification numbers assigned to Taiwanese residents. Cancer cases were identified from histological discharge forms and oncology reports and were classified according to ICD-9 (international classification of diseases, ninth revision) codes. During an average of 8.7 years (range 1.0-12.9 years) of follow-up, 2966587 person years were accumulated, and 9273 cases of incident cancer and 3779 cancer related deaths were recorded.

\section{Statistical analysis}

The primary outcomes of interest were cancer incidence and cancer mortality overall, as well as cancer specific incidence and mortality. We calculated the time to event from the date of enrollment to the date of cancer incident or cancer related death, death due to causes other than cancer, or the end of cohort follow-up (31 December 2008), whichever came first. We used Cox proportional hazards model to estimate hazard ratios and 95\% confidence intervals, adjusting for age (continuous), age squared, sex (male or female), education (middle school or lower, high school, junior college, or college or higher), occupation (white collar, blue collar, self employed, housewife or house husband, or other), smoking status (never, former, or current), pack years of smoking (continuous), alcohol consumption status (never, ever), grams of alcohol/ day (continuous), body mass index (continuous), body mass index squared, physical activity (inactive, low active, medium active, high active, and very high active), and fruit and vegetable intake (continuous). 
Box 1: Diagnostic criteria or categorization of chronic diseases and markers

\section{- Cardiovascular disease markers}

- Blood pressure (Joint National Committee guidelines ${ }^{40}$ )

- Normal: diastolic blood pressure $<80 \mathrm{~mm} \mathrm{Hg}$ and systolic blood pressure $<120 \mathrm{~mm}$ $\mathrm{Hg}$, not receiving medication, and no self reported hypertension

- Prehypertension: (diastolic blood pressure: 80-89 mm Hg or systolic blood pressure: $120-139 \mathrm{~mm} \mathrm{Hg}$ ), not receiving medication, and no self reported hypertension

- Hypertension: diastolic blood pressure $\geq 90 \mathrm{~mm} \mathrm{Hg}$ or systolic blood pressure $\geq 140$ $\mathrm{mm} \mathrm{Hg}$ or receiving medication or self reported hypertension

- Total cholesterol (mmol/L): <4.15, 4.15-4.66, 4.67-5.18, 5.19-6.21, $\geq 6.22$; to convert SI unit of total cholesterol (mmol/L) to $\mathrm{mg} / \mathrm{dL}$ multiply by 38.67

- Heart rate (beats/min): $<70,70-79,80-89, \geq 90$

- Diabetes (WHO criteria ${ }^{41}$ )

- Normal: fasting glucose level $<6.11 \mathrm{mmol} / \mathrm{L}$, not receiving medication, and no self reported diabetes; to convert SI unit of fasting glucose ( $\mathrm{mmol} / \mathrm{L}$ ) to $\mathrm{mg} / \mathrm{dL}$ multiply by 18.02

- Prediabetes: fasting glucose level between 6.11 and $6.95 \mathrm{mmol} / \mathrm{L}$, not receiving medication, and no self reported diabetes

- Diabetes: fasting glucose level $\geq 6.96 \mathrm{mmol} / \mathrm{L}$ or receiving medication or self reported diabetes

- Chronic kidney disease markers (National Kidney Foundation criteria ${ }^{42}$ )

- Proteinuria: no proteinuria, trace proteinuria, proteinuria $(\geq+)$

- Glomerular filtration rate $\left(\mathrm{mL} / \mathrm{min} / 1.73 \mathrm{~m}^{2}\right): \geq 90,60-89,<60$

- Pulmonary disease (Global Initiative for Chronic Obstructive Lung Disease (GOLD) criteria ${ }^{43}$ )

- Normal: forced expiratory volume in one second (FEV)/forced vital capacity (FVC) $\geq 0.70$ and $\mathrm{FEV}_{1} \geq 80 \%$ predicted

- Restrictive: $\mathrm{FEV}_{1} / \mathrm{FVC} \geq 0.70$ and $\mathrm{FEV}_{1}<80 \%$ predicted

- Chronic obstructive pulmonary disease: $\mathrm{FEV}_{1} / \mathrm{FVC}<0.70$

- Gouty arthritis marker

- Uric acid ( $\mu \mathrm{mol} / \mathrm{L})$ : $<297,297-356,357-415,416-475, \geq 476$; to convert SI unit ofuric acid ( $\mu \mathrm{mol} / \mathrm{L}$ ) to $\mathrm{mg} / \mathrm{dL}$ divide by 59.48

We performed multiple imputation with 10 iterations for missing data using Markov Chain Monte Carlo procedures, which assume that all the variables in the imputation model have a joint multivariate normal distribution, and analyses were performed on the dataset with imputed data.

Since chronic diseases or conditions are typically clustered and the association of individual chronic disease or marker with cancer risk tends to be modest, we created two composite scores to investigate the joint effect of the eight chronic diseases and markers: one for risk of incident cancer and another for risk of cancer death. The composite score was calculated in four steps following the procedure by Sullivan et $\left.\mathrm{al}^{44}: 1\right)$ run a multivariable Cox regression model that includes all potential confounders and the eight chronic diseases or markers to estimate the effect of each individual disease or marker independent of potential confounders and other chronic diseases or markers; 2) calculate the individual risk point for each response category or level of a chronic disease or marker variable by dividing the respective regression coefficient with a single constant, which represents the regression coefficient for a one year increase in age in relation to risk of incident cancer or cancer death; 3) round the risk points to the nearest integers; and 4) calculate the composite score by summing the individual risk point for each chronic disease or marker across all eight chronic diseases and markers for each study participant. Therefore, the risk point (ie, weight) given to each condition was proportional to the strength of association (ie, hazard ratio or $\beta$ coefficient from Cox regression model) with risk of incident cancer or cancer death. Supplementary table S1 shows more details on the development of composite chronic disease risk scores and two hypothetical examples.

Using Chiang's life table method, years of life lost were the difference in life expectancy for those with and those without each condition or lifestyle risk factor. ${ }^{45}$ The calculated life expectancy referred to the average remaining years of life expected for a 30 year old. To quantify the contribution of chronic diseases or markers to cancer incidence and cancer mortality, we calculated the population attributable fraction, which is the estimated proportional reduction in cancer incidence or cancer mortality that would occur if chronic diseases or markers were normal. We estimated the population attributable fraction for individual risk factor by the punafcc function in Stata, which was specifically developed for time to event studies. ${ }^{46} \mathrm{We}$ calculated the joint population attributable fraction of clusters of risk factors, which avoids double counting the overlap of multiple risk factors, using the formula shown in the illustration. ${ }^{47} 48$ The relative reduction in risk of incident cancer and cancer death associated with chronic diseases or markers by physical activity was calculated as (hazard ratio inactive $_{\text {-hazard }}$ ratio $_{\text {active }}$ )/ (hazard ratio inactive -1$) \times 100,{ }^{49}$ where hazard ratio inactive is the hazard ratio of chronic diseases or markers among those who were physically inactive, and hazard ratio $_{\text {active }}$ is the hazard ratio among those who were physically active.

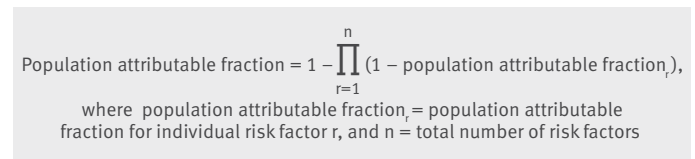

\section{Patient involvement}

This study utilized data from the MJ Health Research Foundation, and details of patient and public involvement are available online (www.mjhrf.org/ main/index/en). Patients, service users, carers, and lay people were not involved in the design, recruitment, or conduct of this study, and outcome measures were not informed by patients' priorities, experience, and preferences. The MJ Health Research Foundation will disseminate all key findings from this study on its website. Participants were thanked in the acknowledgment section.

\section{Results}

Individual associations of chronic diseases with cancer incidence and cancer mortality

Table 1 shows the selected characteristics of the 405878 cohort participants at enrollment. A statistically significantly increased risk of incident cancer was observed for the eight diseases and markers 


\begin{tabular}{|c|c|c|}
\hline Characteristics & Men $(n=194686)$ & Women $(n=211192)$ \\
\hline Mean (SD) age (years) & $40.5(13.8)$ & $40.3(13.8)$ \\
\hline \multicolumn{3}{|l|}{ Education: } \\
\hline Middle school or lower & $38018(19.5)$ & $64861(30.7)$ \\
\hline High school & $44352(22.8)$ & $51927(24.6)$ \\
\hline Junior college & $43400(22.3)$ & $40711(19.3)$ \\
\hline College or higher & $60628(31.1)$ & $44710(21.2)$ \\
\hline Missing & $8288(4.3)$ & $8983(4.3)$ \\
\hline \multicolumn{3}{|l|}{ Occupation: } \\
\hline White collar & $91447(47.0)$ & $83016(39.3)$ \\
\hline Blue collar & $48533(24.9)$ & $16634(7.9)$ \\
\hline Self employed & $17841(9.2)$ & $14878(7.0)$ \\
\hline House wife, house husband & $1799(0.9)$ & $65006(30.8)$ \\
\hline Other & $23078(11.9)$ & $18301(8.7)$ \\
\hline Missing & $11988(6.2)$ & $13357(6.3)$ \\
\hline \multicolumn{3}{|l|}{ Smoking status: } \\
\hline Never & $88309(45.4)$ & $176623(83.3)$ \\
\hline Former & $19881(10.2)$ & $3119(1.5)$ \\
\hline Current & $75815(38.9)$ & $12836(6.1)$ \\
\hline Missing & $10681(5.5)$ & $18614(8.8)$ \\
\hline Mean (SD) pack years* & $17.2(17.5)$ & $7.4(11.8)$ \\
\hline \multicolumn{3}{|l|}{ Alcohol consumption: } \\
\hline Never & $117372(60.3)$ & $173433(82.2)$ \\
\hline Former & $8512(4.4)$ & $2628(1.2)$ \\
\hline Current & $56931(29.2)$ & $13531(6.4)$ \\
\hline Missing & $11871(6.1)$ & $21600(10.2)$ \\
\hline Mean (SD) grams of alcohol/dayt & $9.5(11.5)$ & $6.2(10.8)$ \\
\hline \multicolumn{3}{|l|}{ Physical activity: } \\
\hline Inactive & $93620(48.1)$ & $125273(59.3)$ \\
\hline Low active & $42869(22.0)$ & $44970(21.3)$ \\
\hline Medium active & $30924(15.9)$ & $24128(11.4)$ \\
\hline High active & $13717(7.1)$ & $9916(4.7)$ \\
\hline Very high active & $13516(6.9)$ & $6896(3.3)$ \\
\hline Missing & $40(0.02)$ & $11(0.01)$ \\
\hline Mean (SD) body mass index $\neq$ & $23.8(3.4)$ & $22.2(3.6)$ \\
\hline $\begin{array}{l}\text { Mean (SD) fruit and vegetable } \\
\text { intake§ (portions/day) }\end{array}$ & $5.1(2.8)$ & $5.2(2.7)$ \\
\hline $\begin{array}{l}\text { *Among ever smokers and missing for } \\
\text { †Among ever drinkers and missing for } \\
\text { ¥Missing for } 952(0.2 \%) \text { participants. } \\
\text { §Missing for } 14017(3.5 \%) \text { participan }\end{array}$ & $\begin{array}{l}\text { participants. } \\
\text { p) participants. }\end{array}$ & \\
\hline
\end{tabular}

individually, except blood pressure and pulmonary disease (fig 1). Specifically, total cholesterol level was inversely associated with risk of incident cancer in a dose-response manner, with a 44\% (hazard ratio $1.44,95 \%$ confidence interval 1.33 to 1.56 ; $\mathrm{P}<0.001)$ increase in the lowest level compared with highest level $(<4.15 \mathrm{v} \geq 6.22 \mathrm{mmol} / \mathrm{L})$. In contrast, risk increased with heart rate, with $9 \%$ excess risk (1.09, 1.01 to $1.18 ; \mathrm{P}=0.03)$ when the highest level of heart rate was compared with the lowest level ( $\geq 90 \mathrm{v}<70$ beats/min). Risk increased with severity of proteinuria: $12 \%(1.12,1.04$ to $1.21 ; \mathrm{P}=0.004)$ with trace proteinuria and $21 \%(1.21,1.09$ to 1.35 ; $\mathrm{P}<0.001)$ with proteinuria. A U-shaped association was found between glomerular filtration rate and cancer incidence; compared with a glomerular filtration rate of $60-89 \mathrm{~mL} / \mathrm{min} / 1.73 \mathrm{~m}^{2}$, the risk for those with a low glomerular filtration rate increased by $11 \%(1.11,1.04$ to $1.18 ; \mathrm{P}<0.001)$ and for those with a high glomerular filtration rate by $10 \%(1.10$, 1.04 to 1.16; $\mathrm{P}<0.001)$. Risk increased by $15 \%$ (1.15, 1.07 to $1.24 ; \mathrm{P}<0.001)$ with low uric acid levels $(<297$ $\mu \mathrm{mol} / \mathrm{L})$ compared with a uric acid level between 416 and $475 \mu \mathrm{mol} / \mathrm{L}$. All eight diseases or markers were statistically significantly associated with risk of cancer mortality (fig 2), and the associations were generally stronger than those for cancer incidence.

We conducted multiple sensitivity analyses. First, we conducted another round of multiple imputation with annul income, which was missing for $48.9 \%$ of the study population, and further adjusted for annual income. The results (see supplementary table S2) were almost identical to those not adjusted for annual income (fig 1 and fig 2). Second, we stratified the analysis by years of follow-up ( $<5 v \geq 5$ years) and found similar results across the two stratums (see supplementary table S3). Third, the inverse association of total cholesterol with risk of incident cancer and cancer death was sustained after further exclusion of cancer cases developed within 2-10 years of follow-up (see supplementary table S4) and further adjustment for aspartate transaminase, alanine transaminase, $\alpha$ fetoprotein, and hepatitis B virus infection status (see supplementary table S5). Also, we conducted stratified analyses by smoking status (never versus ever, see supplementary table S6), alcohol consumption status (never versus ever, see supplementary table S7), body mass index $(<25$ $V \geq 25$, see supplementary table S8), physical activity (inactive versus active, see supplementary table S9), and fruit and vegetable intake (low versus high, see supplementary table S10), and associations generally were sustained within each stratum.

Among $177430 \quad(43.7 \%$ of the overall study population of 405878 ) participants who had a second visit, we investigated the associations between chronic diseases and markers at the second visit and risk of incident cancer and cancer death adjusted for updated covariates at the second visit (see supplementary table S11), and the associations were similar to those observed with baseline measurements. We compared the host characteristics of those who had a second follow-up visit with those of the overall study population, and they were comparable (see supplementary table S12).

\section{Joint associations of chronic diseases with cancer incidence and cancer mortality}

Figures 1 and 2 also show the fully adjusted hazard ratios (ie, when all chronic diseases or markers were included in one model in addition to the covariates) among 274818 (67.7\% of the overall study population of 405878) participants who had complete data on all eight chronic diseases or markers, and the host characteristics were comparable to those of the overall study population (see supplementary table S12). The findings, albeit attenuated for some chronic diseases and markers, were generally similar to those adjusted only for covariates.

The individual risk points for each level of the eight chronic disease and marker variables also were presented, which ranged from zero to eight (figs 1 and 2). Total cholesterol level and proteinuria had the highest risk points. The composite chronic disease risk scores were positively associated with cancer incidence 


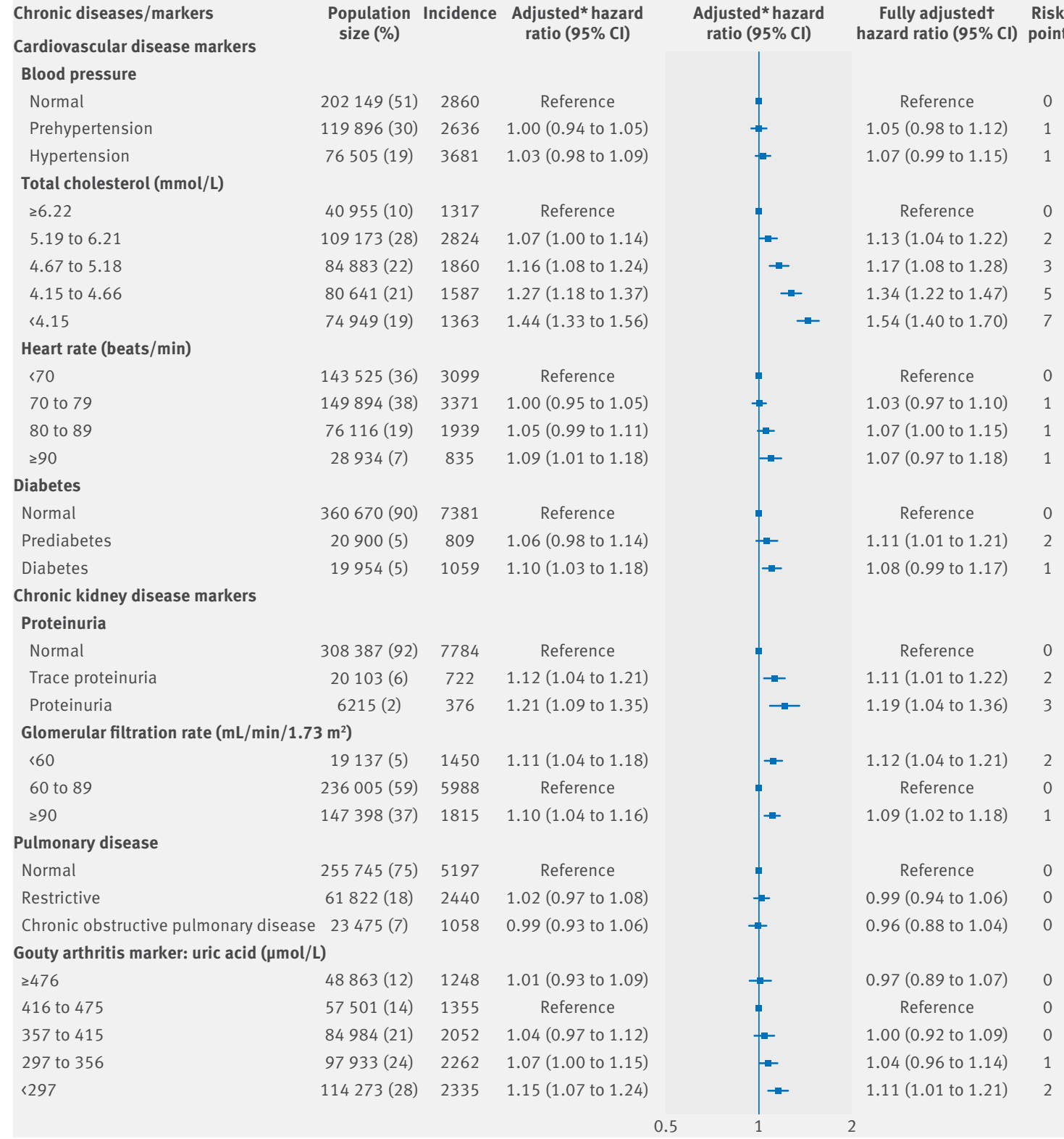

Fig 1 | Chronic diseases and markers and risk of incident cancer. *Adjusted for age (continuous), age squared, sex, education (middle school or lower, high school, junior college, or college or higher), occupation (white collar, blue collar, self employed, housewife or house husband, or other), smoking status (never, former, or current), pack years of smoking (continuous), alcohol consumption status (never, ever), grams of alcohol/day (continuous), body mass index (continuous), body mass index squared, physical activity (inactive, low active, medium active, high active, and very high active), and fruit and vegetable intake (continuous). †All chronic diseases and markers were simultaneously included in the model in addition to the covariates

(fig 3) and cancer mortality (fig 3) in a dose-response manner $(\mathrm{P}<0.001$ for trend for both) in the overall population (red broken lines in figure). Compared with participants scoring $\leq 1$, those who scored 2 to 4,5 to 8,9 to 11 , or $\geq 12$ had an increased hazard ratio for cancer incidence of $1.13,1.36,1.62$, or 2.21 (fig 3), respectively. Similar but stronger associations were observed for cancer mortality; compared with participants scoring $\leq 2$, those who scored 3 to 6,7 to 11,12 to 14 , or $\geq 15$ had an increased hazard ratio of $1.60,2.13,3.04$, or 4.00 for cancer mortality (fig 3), respectively. Also, we applied the risk scores developed at baseline to chronic disease or marker measurements at the second visit among the 177430 participants who had a second visit, and a dose-response relation was also seen for the analysis using patients with data from a second visit (see supplementary table S13).

Every increment of five chronic disease risk scores was associated with a 35\% (hazard ratio $1.35,1.28$ to $1.41 ; \mathrm{P}<0.001)$ increase in overall risk of incident cancer and $50 \%(1.50,1.42$ to 1.58 ; $\mathrm{P}<0.001$ ) increase in overall risk of cancer death (fig 4). For specific cancers, the highest risk of incident cancer was observed for liver cancer followed by 


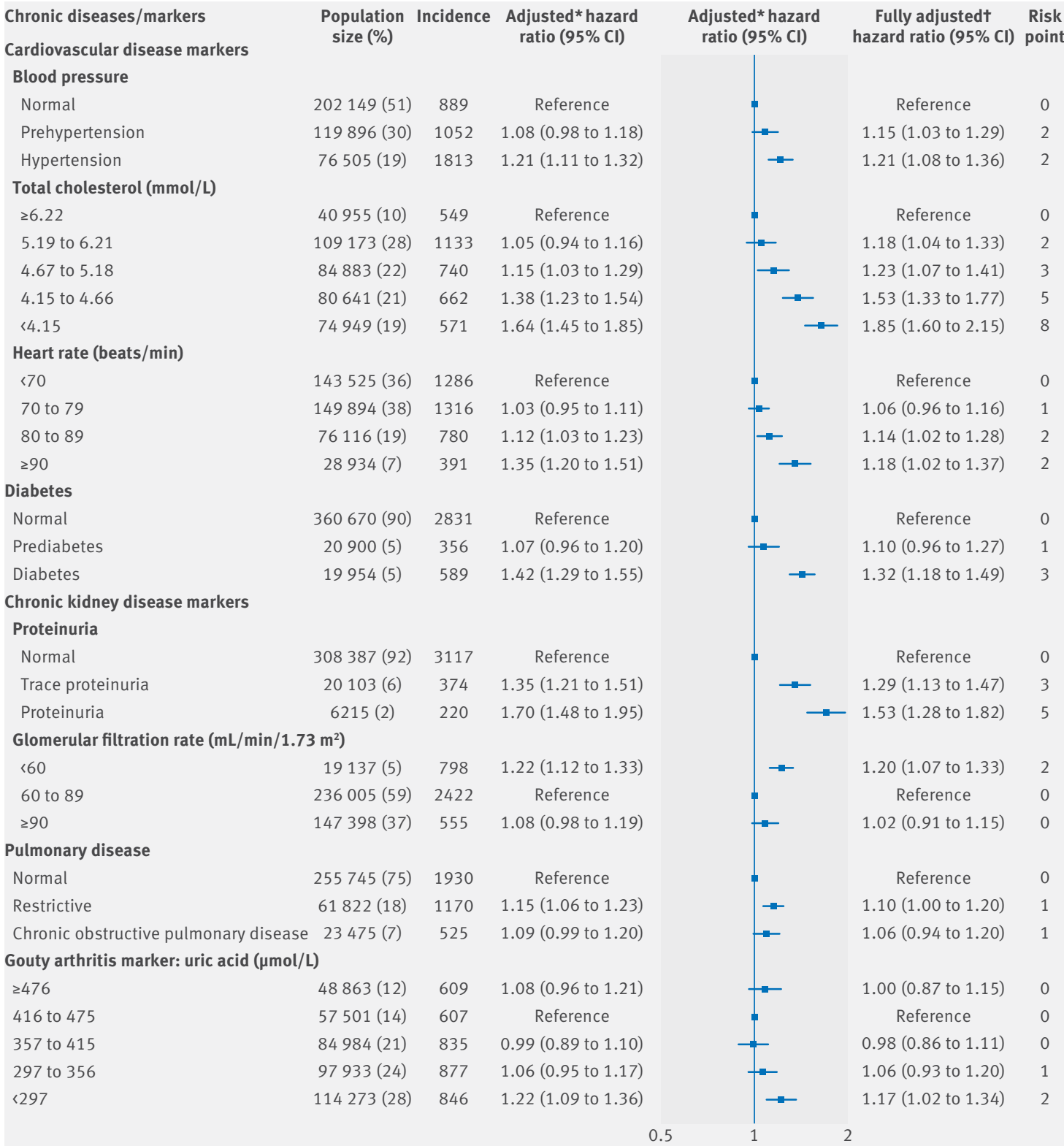

Fig 2 | Chronic diseases and markers and risk of cancer death. *Adjusted for age (continuous), age squared, sex, education (middle school or lower, high school, junior college, or college or higher), occupation (white collar, blue collar, self employed, housewife or house husband, or other), smoking status (never, former, or current), pack years of smoking (continuous), alcohol consumption status (never, ever), grams of alcohol/day (continuous), body mass index (continuous), body mass index squared, physical activity (inactive, low active, medium active, high active, and very high active), and fruit and vegetable intake (continuous). $†$ All chronic diseases and markers were simultaneously included in the model in addition to the covariates

bladder cancer, kidney cancer, oral cancer, and stomach cancer; the highest risk of cancer death was observed for bladder cancer followed by liver cancer, kidney cancer, oral cancer, and colorectal cancer.

\section{Life expectancy}

Higher chronic disease risk scores were associated with greater years of life lost, with the highest scores associated with 13.3 years of life lost in men and 15.9 years of life lost in women (fig 5). Among men, the greatest years of life lost was associated with heart rate $\geq 90$ beats $/$ min ( 7.4 years) followed by trace proteinuria or proteinuria (6.6 years), pulmonary disease (5.8 years), diabetes (5.0 years), glomerular filtration rate $<60 \mathrm{~mL} /$ $\mathrm{min} / 1.73 \mathrm{~m}^{2}$ (3.3 years), hypertension (3.1 years), total cholesterol $<4.15 \mathrm{mmol} / \mathrm{L}$ ( 2.6 years), and uric acid $<297 \mu \mathrm{mol} / \mathrm{L}$ (1.5 years). Among women, the greatest years of life lost was associated with trace proteinuria or proteinuria (6.9 years) followed by diabetes ( 5.9 years), glomerular filtration rate $<60$ $\mathrm{mL} / \mathrm{min} / 1.73 \mathrm{~m}^{2}$ (5.7 years), heart rate $\geq 90$ beats/ $\min$ (5.4 years), total cholesterol $<4.15 \mathrm{mmol} / \mathrm{L}$ ( 4.6 

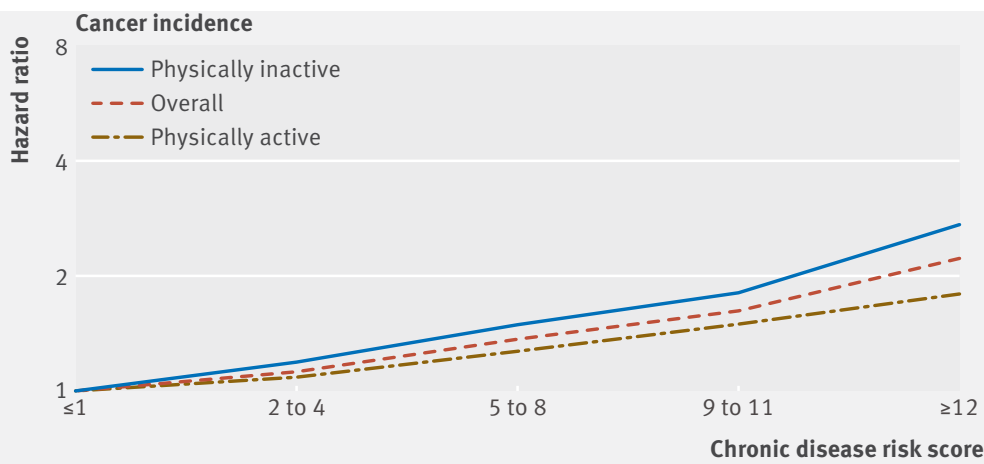

Physically inactive $1.18(0.90$ to 1.56$) \quad 1.49(1.14$ to 1.96$) \quad 1.79(1.35$ to 2.37$) \quad 2.72$ (1.96 to 3.76$)$ Overall 1.13 (0.94 to 1.34$) \quad 1.36(1.14$ to 1.62$) \quad 1.62$ (1.35 to 1.94$) \quad 2.21$ (1.77 to 2.75$)$ Physically active $\quad 1.08(0.86$ to 1.36$) \quad 1.26(1.01$ to 1.59$) \quad 1.49(1.17$ to 1.89$) \quad 1.79$ (1.32 to 2.44$)$ Risk reduction by physical activity (\%)

54

47

38

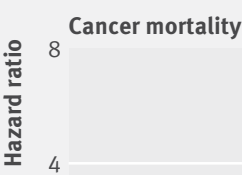

Overall

Physically active

Risk reduction by physical activity (\%)

$1.56(1.03$

24

24
12 to 14

$\geq 15$

Chronic disease risk score

Fig 3 | Chronic disease risk score and risk of incident cancer and cancer death overall and stratified by physical activity. Numbers at bottom of graphs represent adjusted hazard ratios ( $95 \%$ confidence intervals) adjusted for age (continuous), age squared, sex, education (middle school or lower, high school, junior college, or college or higher), occupation (white collar, blue collar, self employed, housewife or house husband, or other), smoking status (never, former, or current), pack years of smoking (continuous), alcohol consumption status (never, ever), grams of alcohol/ day (continuous), body mass index (continuous), body mass index squared, physical activity (inactive, low active, medium active, high active, and very high active), and fruit and vegetable intake (continuous), wherever appropriate. Physically inactive: $<3.75$ metabolic equivalent (MET) hours/week; physically active: $\geq 3.75$ MET hours/week. The relative reduction in risk of incident cancer and cancer death associated with chronic diseases or disease markers by physical activity was calculated as (hazard ratio inactive $^{-}$

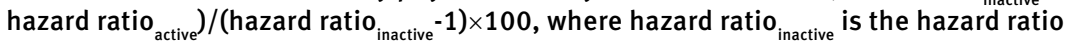
of chronic diseases/markers among those who were physically inactive, and hazard ratio $_{\text {active }}$ is the hazard ratio among those who were physically active
Population attributable fraction of cancer incidence and cancer mortality

Among the eight chronic diseases or markers, low total cholesterol level contributed the most and accounted for $5.7 \%$ of cancer incidence (fig 6). The three cardiovascular disease markers (low total cholesterol level, hypertension or prehypertension, and high heart rate) together accounted for $12.4 \%$, chronic kidney disease markers (trace proteinuria or proteinuria and abnormal glomerular filtration rate combined) for 4.4\%, abnormal uric acid for $3.0 \%$, diabetes or prediabetes for $1.7 \%$, and pulmonary disease for $0 \%$. The total contribution to cancer incidence from the eight chronic diseases or markers (population attributable fraction 20.5\%) was close to that of five major lifestyle factors (24.8\%), including ever smoking, insufficient physical activity, insufficient fruit and vegetable intake, ever alcohol consumption, and non-ideal body mass index. The total contribution to cancer mortality (fig 7) from the eight chronic diseases or markers (population attributable fraction $38.9 \%$ ) was also close to that of five major lifestyle factors $(39.7 \%)$.

\section{Physical activity for reduction of cancer incidence and cancer mortality associated with chronic diseases}

We assessed whether physical activity could reduce the risk of incident cancer or cancer death associated with chronic diseases and markers (as indicated by the chronic disease risk scores). The positive associations of chronic disease risk scores with cancer incidence and cancer mortality were weaker among those who were physically active compared with those who were physically inactive (fig 3). Physical activity attenuated the increased hazards by $38 \%$ to $54 \%$ (an average of $48 \%$ ) for cancer incidence and $14 \%$ to $39 \%$ (an average of $27 \%$ ) for cancer mortality.

\section{Discussion}

In this large cohort study, we found that cardiovascular disease markers, diabetes, chronic kidney disease markers, pulmonary disease, and gouty arthritis marker were individually associated with risk of incident cancer or cancer death. Higher chronic disease risk scores based on these diseases or markers were linked with an increased risk of incident cancer and cancer death in a dose-response manner, with the highest level associated with a more than twofold increase in risk of incident cancer and a fourfold increase in risk of cancer death. Jointly, they accounted for more than one fifth of all incident cancers and more than one third of all cancer deaths, which was comparable to the contribution of five major lifestyle risk factors combined. Physical activity was associated with a nearly $40 \%$ reduction in the excess risks of incident cancer and cancer death associated with chronic diseases and markers.

Chronic diseases are not targeted in current cancer prevention strategies ${ }^{5-7}$; nevertheless, our study revealed a previously overlooked yet substantial impact 


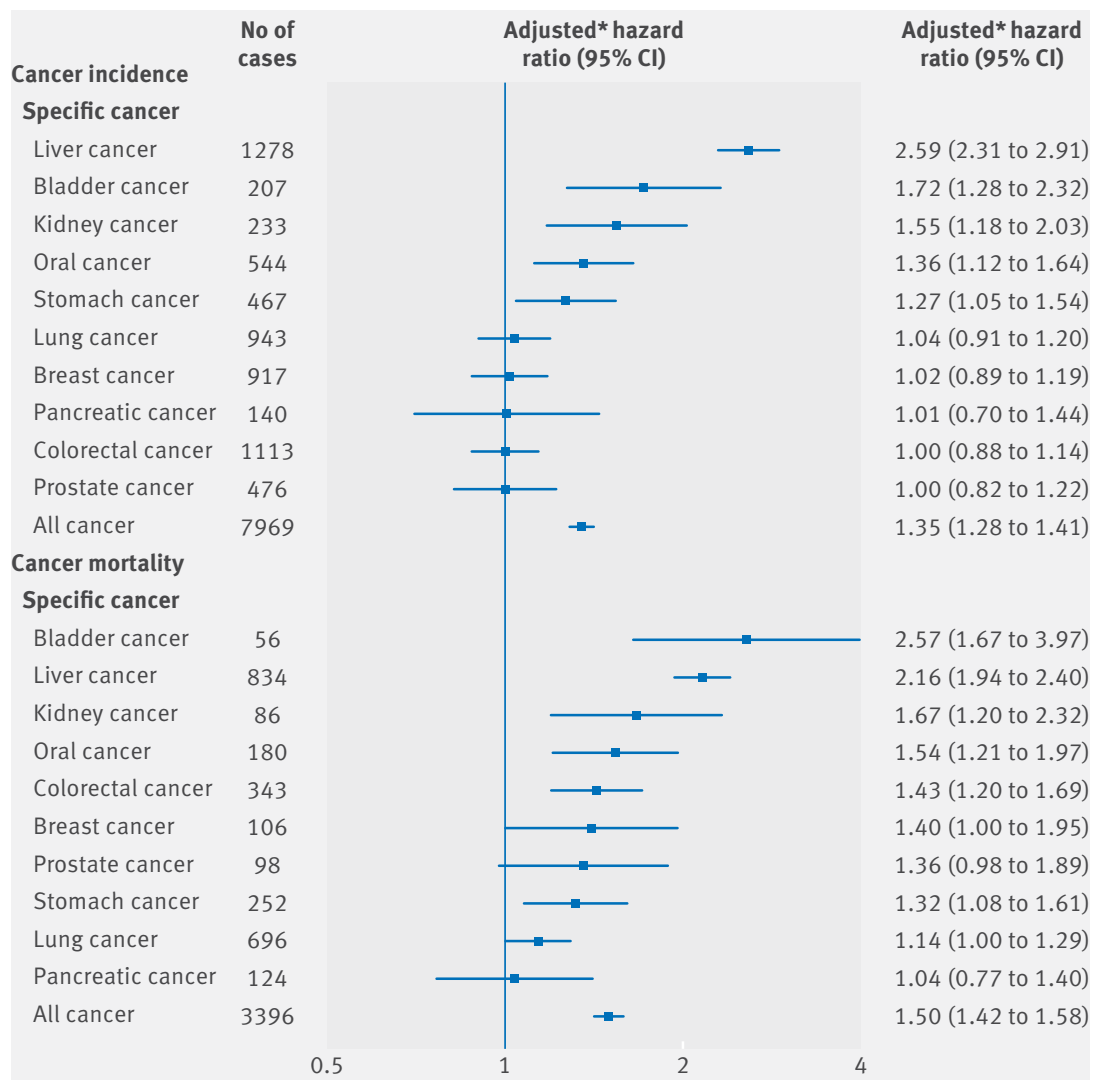

Fig 4 | Adjusted hazard ratios associated every increment of five chronic disease risk scores for cancer incidence and cancer mortality of overall cancers and specific cancers. *Hazard ratios were adjusted for age (continuous), age squared, sex, education (middle school or lower, high school, junior college, or college or higher), occupation (white collar, blue collar, self employed, housewife or house husband, or other), smoking status (never, former, or current), pack years of smoking (continuous), alcohol consumption status (never, ever), grams of alcohol/day (continuous), body mass index (continuous), body mass index squared, physical activity (inactive, low active, medium active, high active, and very high active), and fruit and vegetable intake (continuous)

of chronic diseases on cancer risk. To quantify the joint contribution of the eight chronic diseases or markers to cancer risk and to estimate the proportional reduction in cancer risk that would occur if the eight diseases or markers were reduced to ideal levels, we calculated the population attributable fractions of cancer incidence and cancer mortality associated with the eight chronic diseases and markers. We found that they jointly contributed to more than one fifth (population attributable fraction 20.5\%) of cancer incidence and more than one third of cancer mortality (38.9\%), equally important as five major lifestyle factors, including ever smoking, insufficient physical activity, insufficient fruit and vegetable intake, ever alcohol consumption, and obesity. To date, three studies have estimated the population attributable fraction for diabetes in relation to overall cancer or specific cancers, which ranged from $1.4 \%$ for overall cancer incidence to $7.3 \%$ for pancreatic cancer mortality. ${ }^{51-53}$ Population attributable fraction estimates for other diseases and markers are not available. Even if they were available, the joint contribution, by simply summing the population attributable fraction estimate of each disease or marker from individual studies, could be overestimated owing to the correlations among the diseases and markers.

In our study, we weighted eight chronic diseases or markers into chronic disease risk scores and found that those with higher risk scores were at substantially increased risk of incident cancer and cancer death in a dose-response manner. Among the eight diseases and markers, total cholesterol level and proteinuria carried the most weight in the computation of the overall chronic disease risk scores, suggesting that these two markers are stronger predictors of cancer risk than are the other diseases or markers. Also, we found that the risks of liver, bladder, kidney, stomach, oral, and colorectal cancers were increased with increased chronic disease risk scores, suggesting that chronic diseases or underlying biological alterations may play an important role in the cause of these specific cancers.

The association between total cholesterol level and cancer risk has been controversial. ${ }^{54}$ Based on the relatively well established association between high cholesterol levels and cardiovascular disease risk, we may extrapolate that a high cholesterol level is also linked with cancer risk. However, the association between cholesterol level and cancer risk is less established. Our study showed that those with low cholesterol levels are at higher risk of cancer. Similar findings were also observed in two Korean cohorts $^{18}{ }^{23}$ and a Japanese cohort, ${ }^{19}$ and in white populations. ${ }^{20-22}$ More evidence was needed to clarify whether the inverse association was causal or related to undiagnosed cancer or liver disease, which can lower total cholesterol levels. In our study, the inverse association was sustained after exclusion of cancer cases developed within 10 years of follow-up (see supplementary table S4) and after further adjustment for aspartate transaminase, alanine transaminase, $\alpha$ fetoprotein, and hepatitis B virus infection status (see supplementary table S5). Although we were unable to completely rule out the possibility that a low total cholesterol level is just a marker of risk, the doseresponse relation, the exclusion of cancers during recent follow-up, and further adjustment for liver enzymes, tumor marker, and liver infection minimize the likelihood of reverse causation and lend support for causality.

Our observation of an increased risk associated with low glomerular filtration rate is consistent with previous findings. ${ }^{25-29}$ Our results also suggested that a high glomerular filtration rate $(\geq 90 \mathrm{~mL} /$ $\min / 1.73 \mathrm{~m}^{2}$ ) was associated with an increased risk of incident cancer and cancer death, consistent with a previous finding of a U-shaped association between glomerular filtration rate and cancer. ${ }^{25}$ We also found that cancer incidence and cancer mortality increased substantially with increasing severity of proteinuria in a dose-response manner, which was consistent with the findings from a previous study of overall cancer mortality. ${ }^{55}$ This positive association remained after further adjustment for glomerular filtration rate 


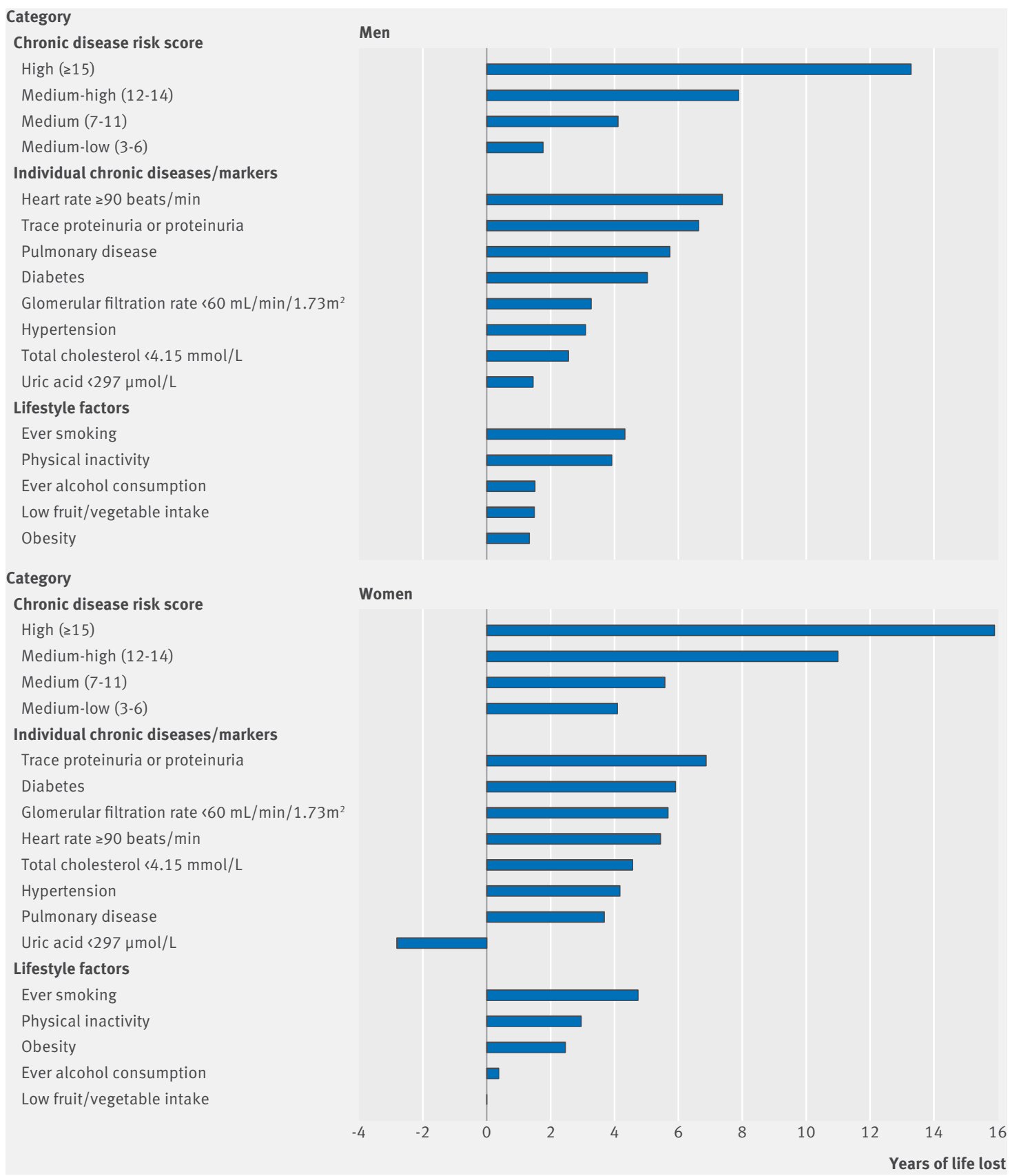

Fig 5 | Years of life lost associated with chronic disease risk score, individual chronic disease or marker, and lifestyle risk factors among men and women. Physically inactivity refers to $\$ 3.75$ metabolic equivalent hours/week, and low fruit and vegetable intake refers to $<2$ portions/day

and other chronic diseases and markers, suggesting that proteinuria is independently associated with cancer risk.

In our previous study, we showed that exercise for as little as 15 minutes a day was associated with a $10 \%$ reduced risk of cancer mortality and that every additional 15 minutes of daily exercise beyond this minimum amount was associated with an additional $1 \%$ reduction. ${ }^{36}$ In the present study, we further found that physical activity was associated with a $48 \%$ reduction in risk of incident cancer and a $27 \%$ reduction in risk of cancer death related to chronic diseases and markers. However, since physical activity was self reported and might be associated with other unmeasured health related behaviors, more studies are warranted to explore how exercise might modify chronic disease related cancer risk independent of other factors.

\section{Implications of findings}

These findings have important implications for cancer prevention. Current global cancer prevention strategies usually focus on modifying lifestyle risk factors. In our study, more than one fifth of incident cancers and 


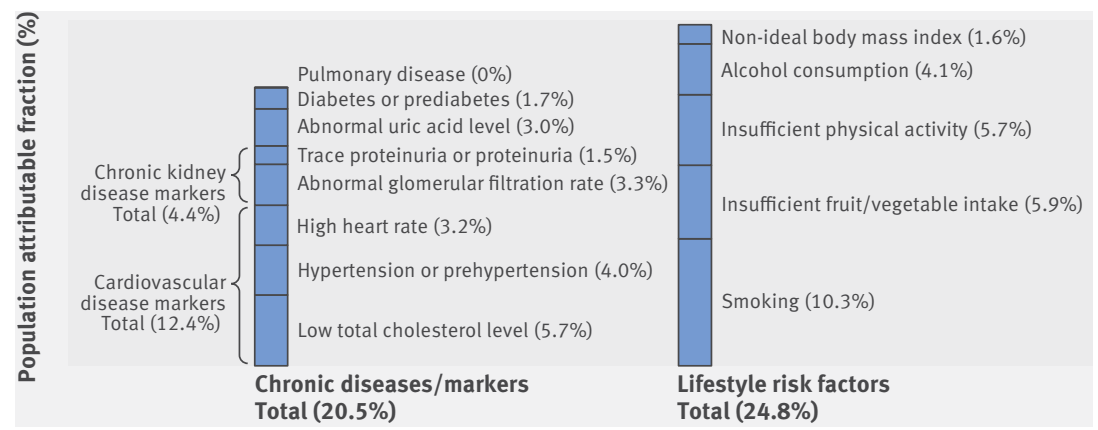

Fig 6 | Population attributable fractions of cancer incidence due to chronic diseases or markers and lifestyle risk factors. Abnormal glomerular filtration rate $=<60$ or $\geq 90$ $\mathrm{mL} / \mathrm{min} / 1.73 \mathrm{~m}^{2}$; abnormal uric acid level $=<416$ or $\geq 476 \mu \mathrm{mol} / \mathrm{L}$; high heart rate $=\geq 70$ beats $/ \mathrm{min}$; low total cholesterol level= $<4.15$ or $4.15-4.66 \mathrm{mmol} / \mathrm{L}$; smoking=ever smoking; insufficient physical activity refers to levels that do not meet current leisure time physical activity recommendation of 150 minutes a week of moderate intensity or 75 minutes a week of vigorous intensity (ie, $<7.50$ metabolic equivalent hours/ week) ${ }^{3639}$; insufficient fruit/vegetable intake refers to $<5$ portions/day recommended by the Word Health Organization ${ }^{50}$; alcohol consumption refers to ever alcohol consumption; non-ideal body mass index refers to body mass index of more than $21.9 \mathrm{~kg} / \mathrm{m}^{2}$, which was the mean body mass index in the normal body mass index $(18.5-24.9)$ category in this study population

more than one third of cancer deaths were attributable to the eight chronic diseases or markers assuming the observed associations are causal, and this was equal to the combined contribution of five lifestyle risk factors. Therefore, a major player will be missed if future cancer prevention strategies do not target non-cancer chronic diseases. At the same time, these findings have important implications for the management of noncancer chronic diseases. Although chronic diseases are responsible for most deaths globally, ineffective management of such diseases in primary care is a global problem and leads to poor health outcomes and increased healthcare costs. ${ }^{56}$ Our results showed that chronic diseases and markers were associated with a substantial reduction in life span that is more

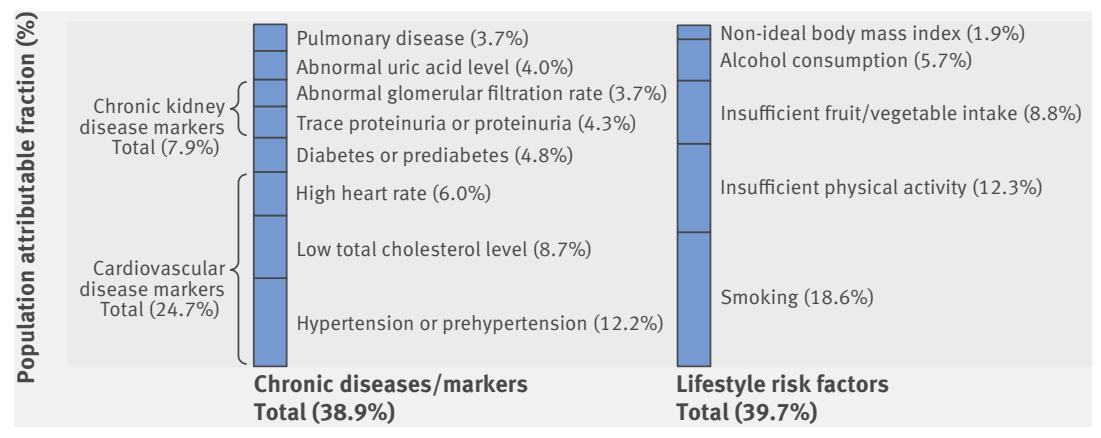

Fig 7 | Population attributable fractions of cancer mortality due to chronic diseases or markers and lifestyle risk factors. Abnormal glomerular filtration rate $=<60$ or $\geq 90 \mathrm{~mL}$ / $\mathrm{min} / 1.73 \mathrm{~m}^{2}$; abnormal uric acid level $=<416$ or $\geq 476 \mu \mathrm{mol} / \mathrm{L}$; high heart rate $=\geq 70$ beats $/$ $\mathrm{min}$; low total cholesterol level= $<4.15$ or $4.15-4.66 \mathrm{mmol} / \mathrm{L}$; smoking=ever smoking; insufficient physical activity refers to levels that do not meet current leisure time physical activity recommendation of 150 minutes a week of moderate intensity or 75 minutes a week of vigorous intensity (ie, $<7.50$ metabolic equivalent hours $/$ week) ${ }^{3639}$; insufficient fruit/vegetable intake refers to $<5$ portions/day recommended by the Word Health Organization ${ }^{50}$; alcohol consumption refers to ever alcohol consumption; non-ideal body mass index refers to body mass index less than or more than $25.6 \mathrm{~kg} / \mathrm{m}^{2}$, which was associated with the lowest risk of cancer mortality in this study intuitive and easier to communicate than commonly used hazard ratios. In addition, our study revealed an overlooked link between chronic diseases and markers and risk of cancer, which is the disease most feared by people. ${ }^{57}{ }^{58}$ Therefore, recognizing the substantial reduction in life span and increase in cancer risk associated with non-cancer chronic diseases could be used as a "teachable moment" to stimulate motivation for better management of such diseases.

\section{Strengths and weaknesses of this study}

Our study has several strengths. First, we examined several major chronic diseases and markers simultaneously, which allowed the estimate of their joint effects on cancer risk. Second, the chronic diseases were diagnosed on the basis of quantitative medical examinations, which allowed for the assessment of the dose-response relation and reduced measurement errors owing to self report. Third, because Taiwan has a central population based death file and a cancer registry, few people were lost to follow-up. Fourth, the increased risk of cancer associated with chronic diseases could result from the shared risk factors between chronic diseases and cancer. For example, physically inactive participants are more likely to have chronic diseases or abnormal disease markers and are also at higher risk of cancer. In our study, we were able to adjust for the major shared risk factors in all statistical models. We also conducted stratified analyses by those risk factors, and the stratified results suggested that the associations between chronic diseases and cancer risks generally remained within each stratum. However, we could not exclude the possibility that some of the observed cancer risk is still due to residual confounding from misclassification of the adjusted confounders, which were self reported or related to unmeasured lifestyle factors that can lead to both the chronic diseases and the cancer. Fifth, we mutually adjusted for the eight chronic diseases or markers, which allowed us to estimate the association between individual chronic diseases and markers and cancer risk independent of other correlated chronic diseases and markers. Finally, we incorporated chronic disease and marker measurements at both baseline and a second visit, which showed similar associations with cancer risk.

Our study had several potential limitations. First, it was an observational study; therefore, the observed associations might not be causal. However, the strength of association, dose-response relation, consistency with previous studies, temporality, and biological plausibility lend support for causality. Second, although the observed associations are expected to be generalizable to other populations, the generalizability of the population attributable fraction estimates also depends on the population specific prevalence of chronic diseases and markers and lifestyle risk factors. Third, we only included the major non-cancer chronic diseases and markers that represent the majority of chronic disease burden, so the total contribution of non-cancer chronic diseases would be greater if 
additional chronic diseases were included. Finally, we used death certificates for determination of cancer mortality, and death certificates in general might not be reliable in differentiating death with cancer from death due to cancer. However, according to a previous study from Taiwan, which assessed the accuracy of underlying cause of death in a systematic sample of 5621 death certificates, the overall accuracy for cancer death was high $(\kappa=0.94)$, suggesting that death certificates on cancer death are reasonably reliable in Taiwan.

\section{Conclusion}

Our study uncovered a substantial impact of chronic diseases jointly on cancer risk, which were equally as important as five lifestyle factors combined. More than one fifth of new cancer cases and more than one third of cancer deaths were attributable to eight common chronic diseases or markers and cancer risk was more substantial when we considered chronic diseases and markers jointly rather than individually. Physical activity is a promising approach to reduce the cancer risk associated with chronic diseases and markers. These findings have important implications for developing new cancer prevention strategies and improving the management of chronic diseases.

We thank the participants. The data used in this research were authorized by and received from MJ Health Research Foundation (authorization code: MJHRFB2014004C). Interpretations and conclusion of this study do not represent the views of MJ Health Research Foundation.

Contributors: HT and CPW contributed equally to this study. XW, CPW, and HT conceived and designed the study. CKT collected, assembled, and quality controlled the data. MH, HT, CPW, XW, SPT, YY, and MKT analyzed and interpreted the data. HT, CPW, and XW drafted the first version of the report. All authors contributed to the content and critical revision of the report and agreed to submit the report for publication. $\mathrm{XW}$ is the guarantor.

Funding: This study was supported by NCI R01 CA170298 to XW, $\mathrm{NCl}$ R01 CA180083 to XW. Center for Transnational and Public Health Genomics at MD Anderson Cancer Center, and in part by Taiwan Ministry of Health and Welfare Clinical Trial Center (MOHW107. TDU-B-212-123004). The funding source had no role in study design, data collection, data analysis, data interpretation, writing of the report, or the decision to submit the paper for publication. XW had full access to all the data in the study and had final responsibility for the decision to submit for publication.

Competing interests: All authors have completed the ICMJE uniform disclosure form at www.icmje.org/coi_disclosure.pdf and declare: no support from any organization for the submitted work; no financial relationships with any organizations that might have an interest in the submitted work in the previous three years; no other relationships or activities that could appear to have influenced the submitted work.

Ethical approval: Informed consent was obtained to authorize data processing and analysis. Ethical reviews were approved by the Institutional Review Boards at the National Health Research Institutes (Taiwan). Individually identifying data were removed and remained anonymous during the entire study.

Data sharing: No additional data available.

Transparency: The lead author (XW) affirms that the manuscript is an honest, accurate, and transparent account of the study being reported; that no important aspects of the study have been omitted; and that any discrepancies from the study as planned (and, if relevant, registered) have been explained.

This is an Open Access article distributed in accordance with the Creative Commons Attribution Non Commercial (CC BY-NC 4.0) license, which permits others to distribute, remix, adapt, build upon this work non-commercially, and license their derivative works on different terms, provided the original work is properly cited and the use is non-commercial. See: http://creativecommons.org/licenses/ by-nc/4.0/.

1 GBD 2015 Mortality and Causes of Death Collaborators. Global, regional, and national life expectancy, all-cause mortality, and cause-specific mortality for 249 causes of death, 1980-2015: a systematic analysis for the Global Burden of Disease Study 2015. Lancet 2016:388:1459-544 doi:10.1016/S01406736(16)31012-1.

2 GBD 2013 Mortality and Causes of Death Collaborators. Global, regional, and national age-sex specific all-cause and cause-specific mortality for 240 causes of death, 1990-2013: a systematic analysis for the Global Burden of Disease Study 2013. Lancet 2015;385:117 71. doi:10.1016/S0140-6736(14)61682-2.

3 Fitzmaurice C, Dicker D, Pain A, et al, Global Burden of Disease Cancer Collaboration. The Global Burden of Cancer 2013. JAMA Oncol 2015;1:505-27. doi:10.1001/jamaoncol.2015.0735.

4 Stewart BW, Kleihues P (Eds). World Cancer Report 2014. IARCPress. Lyon 2014.

5 Cancer Control: Knowledge into Action: WHO Guide for Effective Programmes: Module 2: Prevention. Geneva: World Health Organization 2007

6 World Cancer Research Fund / American Institute for Cancer Research. Food, Nutrition, Physical Activity, and the Prevention of Cancer: a Global Perspective. AICR, 2007.

7 Kushi LH, Doyle C, McCullough M, et al, American Cancer Society 2010 Nutrition and Physical Activity Guidelines Advisory Committee. American Cancer Society Guidelines on nutrition and physical activity for cancer prevention: reducing the risk of cancer with healthy food choices and physical activity. CA Cancer J Clin 2012;62:30-67. doi:10.3322/caac.20140.

8 Emerging Risk Factors Collaboration. Diabetes mellitus, fasting glucose, and risk of cause-specific death. $N$ Engl ) Med 2011;364:829-41. doi:10.1056/NEIMoa1008862.

9 Jee SH, Ohrr H, Sull JW, Yun JE, Ji M, Samet JM. Fasting serum glucose level and cancer risk in Korean men and women. JAMA 2005;293:194-202. doi:10.1001/jama.293.2.194.

10 Giovannucci E, Harlan DM, Archer MC, et al. Diabetes and cancer: a consensus report. CA Cancer J Clin 2010;60:207-21. doi:10.3322/ caac. 20078 .

11 Stocks T, Van Hemelrijck M, Manjer J, et al. Blood pressure and risk of cancer incidence and mortality in the Metabolic Syndrome and Cancer Project. Hypertension 2012;59:802-10. doi:10.1161/ HYPERTENSIONAHA.111.189258.

12 Grossman E, Messerli FH, Boyko V, Goldbourt U. Is there an association between hypertension and cancer mortality? Am J Med 2002;112:479-86. doi:10.1016/S0002-9343(02)01049-5

13 Goon PK, Stonelake PS, Lip GY. Hypertension, anti-hypertensive therapy and neoplasia. Curr Pharm Des 2007;13:2539-44. doi:10.2174/138161207781663073

14 Batty GD, Shipley MJ, Marmot MG, Davey Smith G, Whitehall Study. Blood pressure and site-specific cancer mortality: evidence from the original Whitehall study. Br J Cancer 2003;89:1243-7. doi:10.1038/ sj.bjc.6601255.

15 Stocks T, Bjørge T, Ulmer H, et al. Metabolic risk score and cancer risk: pooled analysis of seven cohorts. Int J Epidemiol 2015;44:1353-63. doi:10.1093/ije/dyv001

16 Wannamethee G, Shaper AG, Macfarlane PW. Heart rate, physical activity, and mortality from cancer and other noncardiovascular diseases. Am J Epidemiol 1993;137:735-48. doi:10.1093/ oxfordjournals.aje.a116734

17 Jouven X, Escolano S, Celermajer D, et al. Heart rate and risk of cance death in healthy men. PLoS One 2011;6:e21310. doi:10.1371/ journal.pone.0021310

18 Kitahara CM, Berrington de González A, Freedman ND, et al. Total cholesterol and cancer risk in a large prospective study in Korea. J Clin Oncol 2011;29:1592-8. doi:10.1200/ ICO.2010.31.5200.

19 Iso H, Ikeda A, Inoue M, Sato S, Tsugane S, JPHC Study Group. Serum cholesterol levels in relation to the incidence of cancer: the JPHC study cohorts. Int / Cancer 2009;125:2679-86. doi:10.1002/ ijc. 24668

20 Sherwin RW, Wentworth DN, Cutler JA, Hulley SB, Kuller LH, Stamler J. Serum cholesterol levels and cancer mortality in 361,662 men screened for the Multiple Risk Factor Intervention Trial. JAMA 1987;257:943-8. doi:10.1001/ jama.1987.03390070063024

21 Schatzkin A, Hoover RN, Taylor PR, et al. Serum cholesterol and cancer in the NHANES I epidemiologic followup study. National Health and Nutrition Examination Survey. Lancet 1987;2:298-301. doi:10.1016/S0140-6736(87)90890-7

22 Strohmaier S, Edlinger M, Manjer J, et al. Total serum cholesterol and cancer incidence in the Metabolic syndrome and Cancer Project (Me-Can). PLoS One 2013;8:e54242. doi:10.1371/journal. pone.0054242. 
23 Song YM, Sung J, Kim JS. Which cholesterol level is related to the lowest mortality in a population with low mean cholesterol level: a 6.4-year follow-up study of 482,472 Korean men. Am J Epidemiol 2000;151:739-47. doi:10.1093/oxfordjournals.aje. a010272

24 Stengel B. Chronic kidney disease and cancer: a troubling connection. J Nephrol 2010;23:253-62

25 Lowrance WT, Ordoñez J, Udaltsova N, Russo P, Go AS. CKD and the risk of incident cancer. I Am Soc Nephrol 2014;25:2327-34. doi:10.1681/ASN.2013060604

26 Wong G, Hayen A, Chapman JR, et al. Association of CKD and cancer risk in older people. J Am Soc Nephrol 2009;20:1341-50. doi:10.1681/ASN.2008090998.

27 Weng PH, Hung KY, Huang HL, Chen JH, Sung PK, Huang KC. Cancerspecific mortality in chronic kidney disease: longitudinal follow-up of a large cohort. Clin J Am Soc Nephrol 2011;6:1121-8. doi:10.2215/ CIN.09011010.

28 Iff S, Craig JC, Turner R, et al. Reduced estimated GFR and cancer mortality. Am J Kidney Dis 2014;63:23-30. doi:10.1053/j. ajkd.2013.07.008.

29 Fried LF, Katz R, Sarnak MJ, et al. Kidney function as a predictor of noncardiovascular mortality. J Am Soc Nephrol 2005;16:3728-35. doi:10.1681/ASN.2005040384.

30 Fini MA, Elias A, Johnson RJ, Wright RM. Contribution of uric acid to cancer risk, recurrence, and mortality. Clin Transl Med 2012;1:16. doi:10.1186/2001-1326-1-16.

31 Taghizadeh N, Vonk JM, Boezen HM. Serum uric acid levels and cancer mortality risk among males in a large general populationbased cohort study. Cancer Causes Control 2014;25:1075-80. doi:10.1007/s10552-014-0408-0.

32 Strasak AM, Rapp K, Hilbe W, et al, VHM\&PP Study Group. Serum uric acid and risk of cancer mortality in a large prospective male cohort. Cancer Causes Control 2007:18:1021-9 doi:10.1007/s10552007-9043-3.

33 Strasak AM, Rapp K, Hilbe W, et al, VHM\&PP Study Group. The role of serum uric acid as an antioxidant protecting against cancer: prospective study in more than 28000 older Austrian women. Ann Oncol 2007;18:1893-7. doi:10.1093/annonc/mdm338.

34 Strasak AM, Lang S, Kneib T, et al, VHM\&PP Study Group. Use of penalized splines in extended Cox-type additive hazard regression to flexibly estimate the effect of time-varying serum uric acid on risk of cancer incidence: a prospective, population-based study in 78,850 men. Ann Epidemiol 2009;19:15-24. doi:10.1016/j. annepidem.2008.08.009.

35 Brenner DR, McLaughlin JR, Hung RJ. Previous lung diseases and lung cancer risk: a systematic review and meta-analysis. PLoS One 2011;6:e17479. doi:10.1371/journal.pone.0017479.

36 Wen CP, Wai JP, Tsai MK, et al. Minimum amount of physical activity for reduced mortality and extended life expectancy: a prospective cohort study. Lancet 2011;378:1244-53. doi:10.1016/S0140 6736(11)60749-6.

37 Wen CP, Cheng TY, Tsai MK, et al. All-cause mortality attributable to chronic kidney disease: a prospective cohort study based on 462 293 adults in Taiwan. Lancet 2008;371:2173-82. doi:10.1016/ S0140-6736(08)60952-6.

38 Wu X, Tsai SP, Tsao CK et al Cohort Profile: The Taiwan MJ Cohort: half a million Chinese with repeated health surveillance data. Int J Epidemiol 2017;46:1744-1744g. doi:10.1093/ije/dyw282.

39 Physical Activity Guidelines Advisory Committee. Physical activity guidelines advisory committee report and 2008 physical activity guidelines for Americans. http://www.health.gov/paguidelines/ committeereport.aspx and http://www.health.gov/paguidelines/pdf/ paguide pdf (accessed November 3, 2017).

40 Chobanian AV, Bakris GL, Black HR, et al, Joint National Committee on Prevention, Detection, Evaluation, and Treatment of High Blood Pressure. National Heart, Lung, and Blood InstituteNational
High Blood Pressure Education Program Coordinating Committee. Seventh report of the Joint National Committee on Prevention, Detection, Evaluation, and Treatment of High Blood Pressure. Hypertension 2003;42:1206-52. doi:10.1161/01. HYP.0000107251.49515.c2.

41 Definition and diagnosis of diabetes mellitus and intermediate hyperglycemia: report of a WHO/IDF consultation. www.who.int/ diabetes/publications/Definition\%20and\%20diagnosis\%20of\%20 diabetes new.pdf

42 Levey AS, Coresh J, Balk E, et al, National Kidney Foundation. National Kidney Foundation practice guidelines for chronic kidney disease: evaluation, classification, and stratification. Ann Intern Med 2003:139:137-47. doi:10.7326/0003-4819-139-2200307150-00013

43 From the Global Strategy for the Diagnosis, Management and Prevention of COPD, Global Initiative for Chronic Obstructive Lung Disease (GOLD). 2015. www.goldcopd.org/.

44 Sullivan LM, Massaro JM, D’Agostino RB Sr. Presentation of multivariate data for clinical use: The Framingham Study risk score functions. Stat Med 2004;23:1631-60. doi:10.1002/sim.1742.

45 Chiang CL. The life table and its applications. RE Krieger Pub Co, 1984.

46 Newson R. Attributable and unattributable risks and fractions and other scenario comparisons. Stata J 2013;13:672-98.

47 Ezzati M, Hoorn SV, Rodgers A, Lopez AD, Mathers CD, Murray CJ, Comparative Risk Assessment Collaborating Group. Estimates of global and regional potential health gains from reducing multiple major risk factors. Lancet 2003;362:271-80. doi:10.1016/S01406736(03)13968-2

48 World Health Organization Global health risks: mortality and burden of disease attributable to selected major risks. World Health Organization, 2009.

49 Birkmeyer JD, Stukel TA, Siewers AE, Goodney PP, Wennberg DE, Lucas FL. Surgeon volume and operative mortality in the United States. N Engl I Med 2003;349:2117-27. doi:10.1056/ NEJMsa035205.

50 Diet, nutrition and the prevention of chronic diseases: report of a Joint WHO/FAO Expert Consultation. WHO Technical Report Series, NO 916. World Health Organization, 2003.

51 Lin CC, Chiang JH, Li Cl, et al. Cancer risks among patients with type 2 diabetes: a 10-year follow-up study of a nationwide population-based cohort in Taiwan. BMC Cancer 2014;14:381. doi:10.1186/1471-2407-14-381.

52 Lam EK, Batty GD, Huxley RR, et al, Asia Pacific Cohort Studies Collaboration. Associations of diabetes mellitus with site-specific cancer mortality in the Asia-Pacific region. Ann Oncol 2011;22:7308. doi:10.1093/annonc/mdq405.

53 Saito E, Charvat H, Goto A, et al. Burden of cancer associated with type 2 diabetes mellitus in Japan, 2010-2030. Cancer Sci 2016:107:521-7. doi:10.1111/cas.12902.

54 Jacobs EJ, Gapstur SM. Cholesterol and cancer: answers and new questions. Cancer Epidemiol Biomarkers Prev 2009;18:2805-6. doi:10.1158/1055-9965.EPI-09-1027.

55 Mok Y, Matsushita K, Sang Y, et al. Association of Kidney Disease Measures with Cause-Specific Mortality: The Korean Heart Study. PLoS One 2016;11:e0153429. doi:10.1371/journal.pone.0153429.

56 Sabaté E, ed. Adherence to Long-Term Therapies: Evidence for Action. Geneva, Switzerland: World Health Organization; 2003

57 Harris Interactive. MetLife Foundation Alzheimer's survey: what America thinks; www.hsph.harvard.edu/news/press-releases/ alzheimers-international-survey/ (retrieved Feb 3, 2017).

58 Blendon RJ, Georges J, et al. Key Findings from a Five-Country Survey of Public Attitudes about Alzheimer's Disease. Poster presented at AAIC, July 2011

Supplementary information: tables S1-S13 